\title{
Physics topics at KLOE-2
}

\author{
Michal Silarski on behalf of the KLOE-2 \\ Collaboration
}

Published online: 28 January 2012

(C) The Author(s) 2012. This article is published with open access at Springerlink.com

\begin{abstract}
The goal of the KLOE-2 experiment operating at the upgraded DA $\Phi$ NE $e^{+} e^{-}$collider is to collect an integrated luminosity of about $20 \mathrm{fb}^{-1}$ over 3-4 years of running. The KLOE-2 apparatus is now equipped with an inner tracker, new scintillation calorimeters and tagging detectors for $\gamma \gamma$ physics. These will allow measurements to refine and extend the KLOE programme on kaon physics and tests of fundamental symmetries as well as quantum interferometry. Here the latest results from the KLOE data analysis are presented and the perspectives at KLOE-2 outlined.
\end{abstract}

Keywords $\mathrm{KLOE}-2 \cdot$ Rare kaon decays $\cdot$ Kaon interferometry $\cdot \mathrm{CP}$ symmetry

\section{Introduction}

In the 2000-2006 data-taking period the KLOE detector, operating at the DAФNE accelerator in the Laboratori Nazionali di Frascati, acquired a total integrated luminosity of $2.5 \mathrm{fb}^{-1}$ at the $\phi$ mass peak (corresponding to about $10^{10} \phi$ decays)

The KLOE- 2 Collaboration: F. Archilli, D. Babusci, D. Badoni, I. Balwierz, G. Bencivenni, C. Bini, C. Bloise, V. Bocci, F. Bossi, P. Branchini, A. Budano, S. A. Bulychjev,

L. Caldeira Balkeståhl, P. Campana, G. Capon, F. Ceradini, P. Ciambrone, E. Czerwiński,

E. Dané, E. De Lucia, G. De Robertis, A. De Santis, G. De Zorzi, A. Di Domenico,

C. Di Donato, D. Domenici, O. Erriquez, G. Fanizzi, G. Felici, S. Fiore, P. Franzini, P. Gauzzi,

G. Giardina, S. Giovannella, F. Gonnella E. Graziani, F. Happacher, B. Höistad, L. Iafolla,

E. Iarocci, M. Jacewicz, T. Johansson, A. Kowalewska, V. Kulikov, A. Kupsc, J. Lee-Franzini,

F. Loddo, G. Mandaglio, M. Martemianov, M. Martini, M. Mascolo, M. Matsyuk, R. Messi,

S. Miscetti, G. Morello, D. Moricciani, P. Moskal, F. Nguyen, A. Passeri, V. Patera,

I. Prado Longhi, A. Ranieri, C. F. Redmer, P. Santangelo, I. Sarra, I. Sarra, B. Sciascia,

A. Sciubba, M. Silarski, C. Taccini, L. Tortora, G. Venanzoni, R. Versaci, W. Wiślicki, M. Wolke, J. Zdebik

M. Silarski $(\bowtie)$

Institute of Physics, Jagiellonian University, 30-059 Cracow, Poland

e-mail: Michal.Silarski@lnf.infn.it 
and $250 \mathrm{pb}^{-1}$ at $\sqrt{s}=1 \mathrm{GeV}$ (giving about $10^{8} \eta$ mesons). This allowed precise studies to be carried out on charged and neutral kaon physics, low energy QCD, as well as tests of CP and CPT conservation [1]. In 2008 the Accelerator Division of the Frascati Laboratory tested a new interaction scheme to allow the beam size to be reduced and the luminosity increased. The test was successful and presently DA $\Phi N E$ can reach a peak luminosity of $5 \times 10^{32} \mathrm{~cm}^{-2} \mathrm{~s}^{-1}$, which is a factor of three better than previously obtained [2]. Following these achievements, the data-taking campaign using the upgraded KLOE detector on the improved machine will start in Autumn 2011. The goal is to collect an integrated luminosity of about $20 \mathrm{fb}^{-1}$ over 3-4 years of running.

\section{The KLOE experiment at DA $\Phi$ NE}

DA $\Phi N E$ is a $e^{+} e^{-}$collider operating near the $\phi$ meson mass peak, at a centre-of-mass energy $\sqrt{s}=1019.45 \mathrm{MeV}$ [1]. The heart of DA $\Phi$ NE consists of two rings in which 120 bunches of electrons and positrons are stored. Electrons are accelerated in the Linac, stored and cooled in the accumulator, and then transferred as a single bunch to the ring. Positrons are first created at an intermediate station in the linac using $250 \mathrm{MeV}$ electrons and then follow the same procedure as for electrons. Electrons and positrons collide with small transverse momenta and produce $\phi$ mesons almost at rest $\left(\beta_{\phi} \approx 0.015\right)$. The $\phi$ decay mainly into $K^{+} K^{-}(49 \%), K_{S} K_{L}(34 \%), \rho \pi(15 \%)$ and $\eta \gamma(1.3 \%)$. The decay products are registered using the KLOE detection setup. This consists of $\mathrm{a} \approx 3.3 \mathrm{~m}$ long cylindrical drift chamber, with a diameter of $\approx 4 \mathrm{~m}$, which is surrounded by the electromagnetic calorimeter. The detectors are placed in the axial magnetic field of a superconducting solenoid of strength $B=0.52 \mathrm{~T}$. The KLOE drift chamber is constructed out of a carbon fibre composite with low- $Z$ and low density and uses a gas mixture of helium (90\%) and isobutane $(10 \%)$. It provides tracking in three dimensions, with a resolution in the transverse plane of about $200 \mu \mathrm{m}$, a resolution in the $z$-coordinate measurement of about $2 \mathrm{~mm}$ and $1 \mathrm{~mm}$ in the decay vertex position. The momentum of a particle is determined from the curvature of its trajectory in the magnetic field with a fractional accuracy $\sigma_{p} / p=0.4 \%$ for polar angles greater than $45^{\circ}$ [1]. The KLOE electromagnetic calorimeter consists of a barrel built out of 24 trapezoidally shaped modules and side detectors (so called endcaps) read out from both sides by a set of photomultipliers [1]. Each of the modules is constructed out of $1 \mathrm{~mm}$ scintillating fibres embedded in $0.5 \mathrm{~mm}$ lead foils to speed up the showering processes. This detector allows measurements of particle energies and flight times with accuracies of $\sigma_{E}=5.7 \% E / \sqrt{E[\mathrm{GeV}]}$ and $\sigma(t)=57 \mathrm{ps} / \sqrt{E[\mathrm{GeV}]} \oplus 140 \mathrm{ps}$, respectively. Analysis of the signal amplitude distributions allows one to determine the place where the particle hit the calorimeter module with accuracy of about $1 \mathrm{~cm}$ in the plane transverse to the fibre direction. The longitudinal coordinate precision is energy dependent: $\sigma_{z}=1.2 \mathrm{~cm} / \sqrt{E[\mathrm{GeV}]}$. Since the $\phi$ mesons are produced almost at rest, kaons arising from the decay move at low speed with their relative angle being close to $180^{\circ}$. As a consequence, the decay products are registered in well separated areas of the detector, which allows identification of $K_{L}$ mesons using reconstructed decays of $K_{S}$ (so called $K_{L}$ tagging) and vice versa. This is a special feature of the DAФNE accelerator which, together with the KLOE detector, is a unique laboratory for kaon physics [3]. 


\section{From KLOE to KLOE-2}

In recent years, a new scheme for the machine based, on Crab-waist optics and a large Piwinsky angle [2], has been proposed to increase the DA $\Phi$ NE luminosity. This has been tested successfully and it has motivated the start of a new KLOE run with an improved detector setup, named KLOE-2. This aims to complete the KLOE physics programme and perform a new set of interesting measurements [3]. For the forthcoming run we have improved the performance of KLOE by adding new sub-detector systems: the tagger system for $\gamma \gamma$ physics studies, the Inner Tracker based on the Cylindrical GEM technology, a tile calorimeter surrounding the inner quadrupoles (QCALT), and a calorimeter between the interaction point (IP) and the first inner quadrupole (CCALT). The tagging system is made up of two different detectors which are already installed and ready for data taking. The Low Energy Tagger (LET) is a small calorimeter placed inside KLOE near the IP, consisting of LYSO crystals read out by silicon photomultipliers. This sub-detector will serve to measure electrons and positrons from $\gamma \gamma$ interactions within a wide energy range centred around $200 \mathrm{MeV}$ with an accuracy $\sigma_{E} \sim 10 \%$. The second tagger, which is called the High Energy Tagger (HET), provides a measurement of the displacement of the scattered leptons with respect to the main orbit. This position detector consists of 30 small BC418 scintillators $3 \times 3 \times 5 \mathrm{~mm}^{3}$, which provide a spatial resolution of $2 \mathrm{~mm}$ (corresponding to a momentum resolution of $\sim 1 \mathrm{MeV} / \mathrm{c}$ ). The output light is collected by light guides with SiPM sensors. The HET allows measurements of particle energies with an accuracy of $\sigma_{E} \sim 2.5 \mathrm{MeV}$ and time with a resolution of $\sigma_{t} \sim 200 \mathrm{ps}$. To improve the acceptance for low momentum tracks, and the vertex reconstruction near the interaction point, we are building the inner tracking chamber. This employs a novel technology with cylindrical GEM (Gas Electron Multiplier) detectors. It will be composed of four concentric layers that will provide 2-D points on a cylinder of known radius. Each layer is a triple-GEM chamber with cathode and anode made of thin polyamide foils. We will also install two additional calorimeters, named QCALT and CCALT. QCALT will be a $1 \mathrm{~m}$ long dodecagonal structure covering the region of the new quadrupoles. It is composed of a sampling of five layers of $5 \mathrm{~mm}$ thick scintillator plates alternated with $3.5 \mathrm{~mm}$ thick tungsten plates, for a total depth of $4.75 \mathrm{~cm}$. The crystal calorimeter CCALT will cover the low polar angle region to increase the acceptance for very forward photons down to $8^{\circ}$. The basic layout consists of two small barrels of LYSO crystals that are read out with APD photosensors. A timing resolution between 300 and 500 ps is expected for $20 \mathrm{MeV}$ photons.

\section{Recent KLOE results and the ongoing analysis in kaon physics}

In addition to the preparation for the first KLOE-2 data-taking period (so-called Step0) and the activities for the design and construction of the new sub-detectors, there are still several ongoing physics analyses of the KLOE data. In kaon physics, apart from the recently published results of a precise $K_{S}$ lifetime measurement [4], there are several studies of neutral kaon interferometry and rare kaon decays, for example $K_{S} K_{L} \rightarrow \pi^{+} \pi^{-} \pi^{+} \pi^{-}$interferometry or the $K_{S} \rightarrow \pi^{0} \pi^{0} \pi^{0}$ branching ratio measurement. These will be described briefly here. The decay of $K_{S}$ meson into three 
pions has not yet been observed, and the best limit on the branching ratio $B R\left(K_{s} \rightarrow\right.$ $\left.3 \pi^{0}\right)<1.2 \times 10^{-7}[5]$ is about two orders of magnitude larger than predictions based on the Standard Model. Moreover, this process violates $C P$ symmetry and, assuming $C P T$ invariance, allows one to investigate direct $C P$ violation. At KLOE this decay is reconstructed by searching for events with a $K_{L}$ interaction in the calorimeter (so called $K_{L}$-crash), six photon clusters and no tracks from the interaction point. The background originates mainly from $K_{S} \rightarrow 2 \pi^{0}$ events with two spurious clusters from splittings or accidental activity or is due to false $K_{L}$-crash tags from $\phi \rightarrow K_{S} K_{L} \rightarrow$ $\pi^{+} \pi^{-} \pi^{0} \pi^{0} \pi^{0}$ events. In the latter case, charged pions from $K_{S}$ decays interact in the low-beta insertion quadrupoles, ultimately simulating the $K_{L}$-crash signal, while $K_{L}$ decays close to the interaction point produce six photons [5]. To reduce the background, we first perform a kinematic fit with 11 constraints: energy and momentum conservation, the kaon mass and the velocities of the six photons. Cutting at a reasonable $\chi^{2}$ value reduces considerably the background from false $K_{L}$-crash events with little signal loss. In order to gain a good rejection of background from events with split or accidental clusters, we look at the correlation between the following two $\chi^{2}$-like discriminating variables, $\chi_{3 \pi}^{2}$ and $\chi_{2 \pi}^{2} \cdot \chi_{3 \pi}^{2}$ is the quadratic sum of the residuals between the nominal $\pi^{0}$ mass and the invariant masses of three photon pairs formed from the six clusters present. $\chi_{2 \pi}^{2}$ is based instead on energy and momentum conservation in the $\phi \rightarrow K_{S} K_{L}, K_{S} \rightarrow \pi^{0} \pi^{0}$ decay hypothesis, as well as on the invariant masses of two photon pairs. Both variables are evaluated with the most favorable cluster pairing in each case [5]. In addition, in order to improve the quality of the photon selection using $\chi_{2 \pi}^{2}$, we cut on the variable $\Delta E=\left(m_{\Phi} c^{2} / 2-\sum E_{\gamma_{i}}\right) / \sigma_{E}$, where $\gamma_{i}$ is the $\mathrm{i}-t h$ photon from the four chosen in the $\chi_{2 \pi}^{2}$ estimator and $\sigma_{E}$ is the appropriate resolution. For $K_{S} \rightarrow 2 \pi^{0}$ decays plus two background clusters, we expect $\Delta E \sim 0$ while, for $K_{S} \rightarrow 3 \pi^{0}, \Delta E \sim m_{\pi^{0}} c^{2} / \sigma_{E}$. At the end of the analysis we cut also on the minimal distance between the photon clusters to refine the rejection of events with split clusters (see left panel of Fig. 1). Applying the preliminary selection cuts, we find zero candidates in $1.7 \mathrm{fb}^{-1}$ of real data with zero events expected from Monte Carlo, corresponding to an effective statistics of two times that of the data. This results in a new preliminary upper limit on the branching ratio $B R\left(K_{S} \rightarrow 3 \pi^{0}\right)<2.9 \times 10^{-8}$, which suggests that a first observation of the decay might be feasible at KLOE-2. A unique feature of the $\Phi$-factory is the production of neutral kaon pairs in a pure quantum state so that we can study quantum interference effects and tag pure monochromatic $K_{S}$ and $K_{L}$ beams. The decay rate of the system, for example, to the $\pi^{+} \pi^{-} \pi^{+} \pi^{-}$final state is proportional to:

$$
I\left(\pi^{+} \pi^{-}, \pi^{+} \pi^{-}, \Delta t\right) \propto e^{-\Gamma_{L} \Delta t}+e^{-\Gamma_{S} \Delta t}-2 e^{-\frac{\Gamma_{L}+\Gamma_{S}}{2} \Delta t} \cos (\Delta m \Delta t),
$$

where $\Delta t$ is the time difference between the decays of the two kaons. If the neutral kaon system evolves in time as a pure quantum state, kaons cannot decay at the same time due to the destructive interference. But there are several potential mechanisms leading to decoherence of the state which may result from fundamental modifications of Quantum Mechanics or CPT violation induced, e.g.,by quantum gravity [3]. Thus, by measuring the $\Delta t$ distribution, we can test the foundations of Quantum Mechanics as well as different phenomenological models of Quantum Gravity. At KLOE, the selection of a $\phi \rightarrow K_{S} K_{L} \rightarrow \pi^{+} \pi^{-} \pi^{+} \pi^{-}$signal requires two vertices, each with two opposite-curvature tracks inside the drift chamber, with invariant mass and 

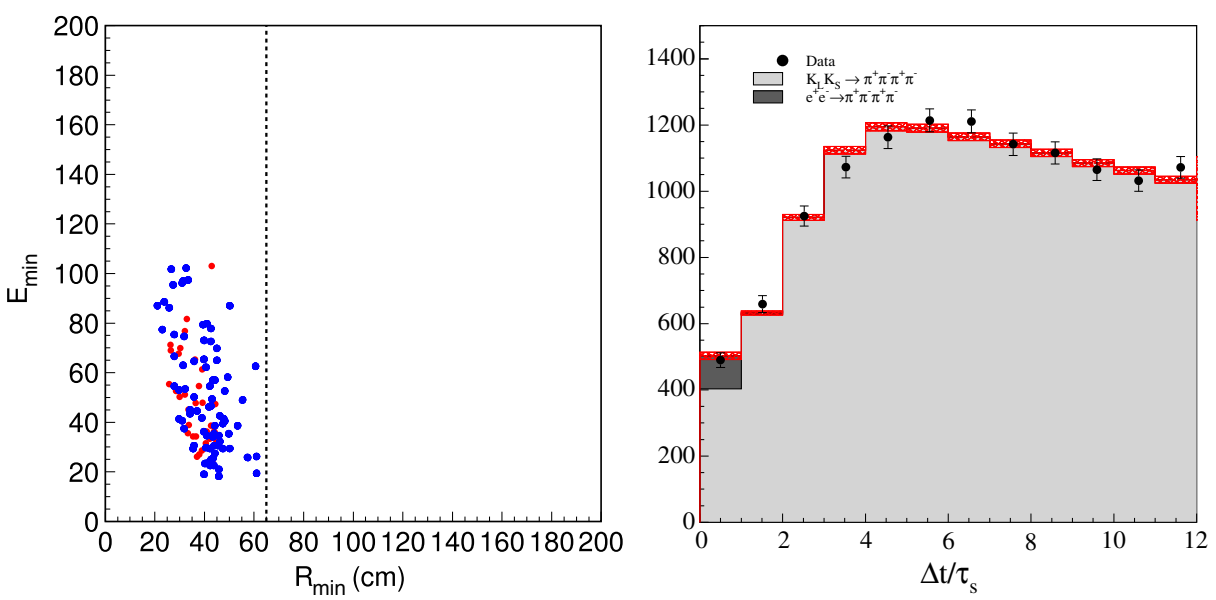

Fig. 1 Left panel The distribution of the minimal energy of the cluster versus minimal distance $\left(R_{\min }\right)$ between clusters in the event for data (red) and MC (blue). The dashed line corresponds to the $R_{\min }$ cut used. Right panel Number of events as a function of the difference in the decay time of the two $\pi^{+} \pi^{-}$vertices in $K_{S}$ lifetime units. The measured $I\left(\pi^{+} \pi^{-}, \pi^{+} \pi^{-}, \Delta t\right)$ distribution is fitted: black points are data and the fit result is shown by the histogram

total momentum compatible with two neutral kaon decays. The resolution in $\Delta t$, the absolute value of the time difference of two $\pi^{+} \pi^{-}$decays, benefits from the precise momentum measurements and from the completeness of the kinematics of the events [6]. The experimental $\Delta t$ distribution is fitted with (1), modified by parameters expressing decoherence in the different models described in [3]. The fit is performed taking into account the resolution and detection efficiency, the background from coherent and incoherent $K_{S}$ regeneration on the beam pipe wall, and the small contamination from the non-resonant $e^{+} e^{-} \rightarrow \pi^{+} \pi^{-} \pi^{+} \pi^{-}$channel. The resulting distributions are shown together with the fit results in Fig. 1. On the basis of the $1.7 \mathrm{fb}^{-1}$ of data, useful estimates could be made of several decoherence and CPT violating parameters. The details of the analysis and the results obtained can be found in [7]. It is worth mentioning that we have almost finished a refined analysis of the KLOE data with several improvements in the methodology. Moreover, KLOE-2 with increased statistics $(\times 4$ in Step0) and improved resolution in the vertex reconstruction $(\times 3$ with the Inner Tracker) will be able to achieve the best experimental sensitivity in some observables, hopefully reaching the level of the Planck scale [3].

\section{Summary}

KLOE is a high precision experiment which allows detailed studies of both kaons and light scalar mesons, as well as tests of the conservation of CP, CPT and low energy QCD. The success of the DA $\Phi$ NE upgrade motivated a new experiment, KLOE-2, which aims at completing and extending the KLOE physics programme. We have started design and construction of new sub-detectors, which will improve the detection performance. The tagging system for $\gamma \gamma$ physics is installed and ready 
for the first phase of the experiment in which we expect to accumulate about $5 \mathrm{fb}^{-1}$. The next data-taking campaign during 2013-15 will be conducted with the Inner Tracker and improved photon acceptance brought about by the calorimeters in the final focusing region. The total integrated luminosity expected in this second phase is about $20 \mathrm{fb}^{-1}$.

Acknowledgement The author would like to express his gratitude to prof. Colin Wilkin for proof reading of the article and many useful comments.

Open Access This article is distributed under the terms of the Creative Commons Attribution License which permits any use, distribution, and reproduction in any medium, provided the original author(s) and the source are credited.

\section{References}

1. Bossi, F., et al.: Precision Kaon and Hadron physics with KLOE. Riv. Nuovo Cim. 31, 531-623 (2008)

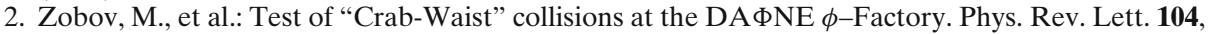
174801-174806 (2010)

3. Amelino-Camelia, G., et al.: Physics with the KLOE-2 experiment at the upgraded DA $\Phi$ NE. Eur. Phys. J. C 68, 619-681 (2010)

4. Ambrosino, F., et al.: Precision measurement of the $K_{S}$ meson lifetime with the KLOE detector. Eur. Phys. J. C 71, 1604-1610 (2011)

5. Ambrosino, F., et al:: A direct search for the CP-violating decay $K_{S} \rightarrow 3 \pi^{0}$ with the KLOE detector at DAФNE. Phys. Lett. B 619, 61-70 (2005)

6. Ambrosino, F., et al.: First observation of quantum interference in the process $\phi \rightarrow K_{S} K_{L} \rightarrow$ $\pi^{+} \pi^{-} \pi^{+} \pi^{-}$: a test of quantum mechanics and CPT symmetry. Phys. Lett. B 642, 315-321 (2006)

7. Di Domenico, A., et al.: Search for CPT violation and decoherence effects in the neutral kaon system. J. Phys. Conf. Ser. 171, 012008-012023 (2009) 